\title{
Philippe Sansonetti and Cellular Microbiology
}

\author{
Javier Pizarro-Cerdá ${ }^{1}$ and Guy Tran Van Nhieu² \\ ${ }^{1}$ Yersinia Research Unit, Department of Microbiology, Institut Pasteur, 75005 Paris, France \\ ${ }^{2}$ Equipe Communication Intercellulaire et Infections Microbiennes, Centre de Recherche Interdisciplinaire en \\ Biologie (CIRB), Collège de France, 75005 Paris, France
}

Philippe Sansonetti is a major international figure in the field of infectious diseases, member of numerous organizations including EMBO, the Royal Society and the French and US National Academies of Sciences. Trained as a medical doctor, PS practiced internal medicine and directed the Institut Pasteur Medical Center while running a research laboratory at the Institut Pasteur. PS has also been actively involved in vaccine development with the World Health Organization.

In the mid-nineties, PS cemented the development of a novel field of investigation, 'cellular microbiology', unraveling the multiple molecular mechanisms that allow the model enteropathogen Shigella flexneri to subvert cellular functions during invasion and colonization of the intestinal mucosa. In more recent years, PS has been a pioneer of our current understanding of the thin line that separates commensals from pathogens within the mammalian gut microbiota. Together with Richard S. Stephens, Philippe J. Sansonetti founded in 1999 the journal Cellular Microbiology, creating a forum for the publication of premier research on interactions between viruses, fungi, parasites, bacteria and their toxins with mammalian cells, allowing the exploration of pathogenic or mutualistic interactions from in vitro systems and animal model perspectives. This 20th anniversary special issue of Cellular Microbiology is a tribute to Philippe Sansonetti, featuring contributions from prominent investigators, including former students, colleagues and close friends and illustrating his broad impact in this exciting and ever expanding field. 